\title{
Effets d'infusions d'un mélange complet d'acides gras volatils en cours de repas sur la prise alimentaire de vaches taries ou en lactation
}

\author{
P Faverdin
}

INRA, Station de Recherches sur la Vache Laitière, St-Gilles, 35590 L'Hermitage, France

\begin{abstract}
Summary - Infusions of 3 or $6 \mathrm{~mol}$ of a volatile fatty acid mixture into the rumen for $3 \mathrm{~h}$ during the morning feeding depress feed intake of lactating dairy cows (1.5 kg of dry matter (DM)) and, to a lesser extent, that of dry cows $(0.8 \mathrm{~kg} D M)$. These differences occur between 1 and $3 \mathrm{~h}$ after the beginning of the meal and are not recovered over the day.
\end{abstract}

Introduction - Les effets dépressifs des infusions d'acides gras volatils (AGV) dans le rumen sur l'ingestion d'aliments ont été souvent observés chez la chèvre (Baile et Forbes, 1974), mais semblent remis en cause à des doses physiologiques (DeJong, 1981; Focant, 1986). Le but de cette étude était de décrire l'évolution du comportement alimentaire au cours de la journée en réponse à l'infusion d'un mélange complet d'AGV à 2 doses physiologiques, en début de grand repas, en fonction du stade physiologique de l'animal (vaches en lactation ou taries).

Matériel et Méthodes - Des infusions dans le rumen de $5 \mathrm{I}$ de solution témoin (eau $=0 \mathrm{M}$ ) ou de 2 doses ( 3 ou $6 \mathrm{~mol}: 3 \mathrm{M}, 6 \mathrm{M}$ ) d'un mélange complet d'AGV $(62 \%$ C2, $21 \%$ C3, $12 \%$ C4, 5\% d'autres AGV) ont été réalisées sur 3 vaches en lactation ( $20 \mathrm{~kg}$ de lait $j$ ) et 3 vaches taries munies de canules du rumen. Chaque groupe de 3 vaches a reçu les 3 traitements suivant un schéma en carré latin $3 \times 3$ (avec 3 ou 4 répétitions dans le temps), dont la durée d'une période était de $24 \mathrm{~h}$. Les vaches en lactation recevaient une ration complète composée d'un mélange de $70 \%$ d'ensilage de maïs et de $30 \%$ d'aliments concentrés alors que seul l'ensilage de maïs était offert aux vaches taries. La ration était distribuée 2 fois par jour à $9 \mathrm{~h} 30$ et $15 \mathrm{~h}$ 30. L'accès à la ration était fermé à partir de 21 $\mathrm{h}$. Les infusions débutaient à $8 \mathrm{~h} 30$ et se poursuivaient jusqu'à 11 h 30 , essentiellement pendant le grand repas du matin. Du bicarbonate de sodium (250 g) était ajouté au repas du matin pour limiter les baisses de $\mathrm{pH}$ dues à l'infusion sous forme acide. Les quantités ingérées ont été enregistrées en continu à l'aide de balances placées sous les auges et reliées à un micro-ordinateur (Faverdin, 1985). Des mesures cinétiques des principaux paramètres du rumen $(\mathrm{pH}$, acidité totale, composition molaire du jus de rumen) ont été effectuées sur les vaches en lactation au cours d'une période de mesure supplémentaire.

Résultats et Discussion - La vitesse d'ingestion des vaches en lactation a été ralentie entre $30 \mathrm{~min}$ et $3 \mathrm{~h}$ après le début du repas par l'infusion du mélange d'AGV. Cette diminution d'appétit a été synchrone ( $1 \mathrm{~h}$ après le début du repas) d'une concentration en AGV et d'une acidité du jus de rumen plus importantes qu'avec l'infusion témoin (tableau 1). Ces différences ont disparu environ $4 \mathrm{~h}$ après la distribution de la ration. L'écart des quantités ingérées cumulées entre les infusions témoin et $6 \mathrm{M}$ s'est accru au cours des 3 premières heures pour atteindre $1,5 \mathrm{~kg}$ de MS (fig 1) et n'a pratiquement plus varié durant le reste de la journée. Chez les vaches taries, les effets des infusions sur la prise alimentaire ont été similaires mais de plus faible amplitude. Les vitesses d'ingestion n'ont été ralenties qu'entre $1 \mathrm{~h}$ et $1 \mathrm{~h} 30$ après le début du repas. L'écart des quantités ingérées cumulées n'a pas 
Tableau I. Effets d'infusions d'AGV sur la prise d'aliments, le pH et la concentration en AGV du jus de rumen à $1 \mathrm{~h}$ et $4 \mathrm{~h} 30$ après le début du repas.

\section{$1 \mathrm{~h}$}

$4 h 30$

\begin{tabular}{lcccccc}
\hline Infusion AGV (mol) & 0 & 3 & 6 & 0 & 3 & 6 \\
Vitesse d'ingestion (g MS/min) & 52 a & $38^{\mathrm{b}}$ & $41^{\mathrm{b}}$ & 14 & 17 & 18 \\
MS ingérées cumulées (kg) & $3,7^{\mathrm{a}}$ & $3,0^{\mathrm{b}}$ & $3,2^{\mathrm{b}}$ & $7,9 \mathrm{a}$ & 6,8 ab & $6,6^{\mathrm{b}}$ \\
pH du jus de rumen & $6,74^{\mathrm{a}}$ & $6,58^{\mathrm{b}}$ & $6,30^{\mathrm{b}}$ & 6,23 & 6,37 & 6,40 \\
AGV totaux du rumen (mmol/) & $72^{\mathrm{a}}$ & $80^{\mathrm{ab}}$ & $93^{\mathrm{b}}$ & 96 & 87 & 86
\end{tabular}

Les moyennes n'étant affectées d'aucune lettre commune sont significativement différentes à $P<0,05$.

dépassé $0,8 \mathrm{~kg}$ de $M S(P<0,1$ seulement) (fig 1). Pour les 2 groupes d'animaux, les différences d'ingestion d'aliments entre les doses 3 et $6 \mathrm{M}$ ont été très faibles. Les consommations d'eau du matin ou du soir, des vaches taries ou en lactation, ont été identiques avec les 3 traitements.

L'infusion de $6 \mathrm{M}$ d'AGV (environ 1300 kcal d'énergie nette (EN)) à des vaches en lactation a provoqué une baisse d'ingestion d'aliment (environ $2000 \mathrm{kcal}$ d'EN) essentiellement pendant l'infusion. Cette baisse n'est pas récupérée au cours de la journée. Les vaches taries ont été moins sensibles aux traitements d'infusion. Cette différence, qui mériterait d'être confirmée, pourrait aussi provenir des rations utilisées (Simkins et al, 1965).

Baile CA, Forbes JM (1974) Physiol Rev 54, 161-214

DeJong $A$ (1981) Thèse de Doctorat, Univ Groningen

Faverdin $P$ (1985) Thèse de Doctorat, Ing Sci Agro, Inst Natl Agron, Paris-Grignon

Focant M (1986) Thèse de Doctorat, Sci Agronom, Université de Louvain

Simkins KL, Suttie JW, Baumgardt BR (1965) J Dairy Sci 48, 1635-1642

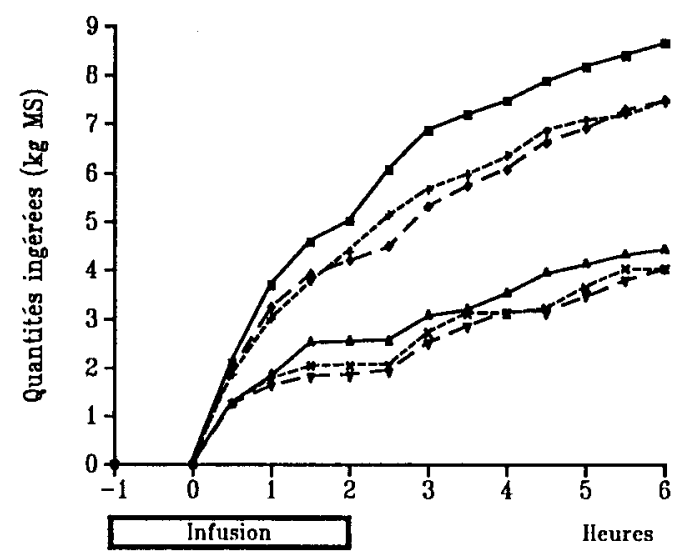

Fig 1. Evolution des quantités de matière sèche (MS) ingérées cumulées sur $6 \mathrm{~h}$ en réponse à une infusion d'eau (-), de $3(--)$ ou de $6 \mathrm{~mol}(--)$ d'un mélange complet d'AGV sur des vaches en lactation $(\mathbf{\square}+\bullet)$ ou taries $(\Delta \times \nabla)$. 